\title{
Disponibilidade Nutricional da Bacia do Pina e Rio Tejipió (Recife- PE- Brasil) EM RELAÇÃo AOS NUTRIENTES E BIOMASSA PRIMÁRIA (SETEMBRO/2000)
}

\author{
FLÁVIA CRISTINA ROCHA DO NASCIMENTO ${ }^{1}$ \\ flaoceano@ig.com.br \\ KÁTIA MUNIZ ${ }^{1}$ \\ FERNANDO ANTÔNIO DO NASCIMENTO FEITOSA ${ }^{1}$ \\ JANAINA PAULINE DE ARAÚJO ${ }^{1}$ \\ ROSSANA MARIA SOARES DA SILVA ${ }^{1}$ \\ GORETTI SÔNIA DA SILVA ${ }^{2}$ \\ MANUEL DE JESUS FLORES MONTES ${ }^{1}$
}

${ }^{1}$ Departamento de Oceanografia da UFPE

${ }^{2}$ Departamento de Zoologia da UNICAP

\begin{abstract}
RESUMO
O presente trabalho foi desenvolvido na Bacia do Pina-PE cuja área estuarina está situada na parte interna do Porto do Recife, em plena zona urbana. É formada pela confluência dos rios Tejipió, Jiquiá, Jordão, Pina e pelo braço sul do Capibaribe. O trabalho teve como objetivo analisar a dinâmica desta bacia em relação à salinidade, determinar o comportamento conservativo ou não conservativo dos nutrientes, registrando a disponibilidade nutricional e o desenvolvimento da população fitoplanctônica através da sua biomassa ao longo do estuário. As amostras de água foram coletadas na superfície para a determinação das análises hidrológicas e biomassa fitoplanctônica e na coluna d'água para a salinidade, utilizando-se garrafa de Nansen, em quatorze estações fixas, durante a baixa-mar, no final do inverno de 2000. Quanto a salinidade a Bacia do Pina apresentouse, nas porções mais externas como um estuário de cunha salina, nas porções intermediárias homogêneo e parcialmente misturado e na porção superior bem misturado. Os nutrientes apresentaram um comportamento não conservativo, com o nitrito, nitrato e o silicato sendo absorvidos (principalmente em salinidades mais baixas) e amônia e o fosfato adicionados ao ecossistema. Em relação ao fosfato, as pequenas variações mostram que o sistema tampão está ocorrendo com esse nutriente. A relação N:P variou de 14:1 a 3:1, demonstrando um ambiente altamente dinâmico quanto à disponibilidade nutricional, sem apresentar, porém nenhum nutriente limitante, apesar dos valores estarem abaixo da relação de Redfield. O nitrogênio foi o fator principal que influenciou o desenvolvimento fitoplanctônico, detectado através da relação N:P e pela análise dos Componentes Principais. O valor máximo do material em suspensão foi de 60,0 $\mathrm{mg} / \mathrm{l}$, considerado alto devido aos intensos aportes continentais. Esse fato propicia uma melhor penetração de luz, facilitando o processo fotossintético em todas as estações, apresentando-se a área como um ambiente eutrófico, com um valor máximo de clorofila de 105,20 mg.m ${ }^{-3}$. De um modo geral, porém, a biomassa aumentou da boca da barra (estação 1 ) até a estação 8 , diminuindo a partir daí até o ponto de confluência dos rios e elevando-se novamente nas estações que se encontram no estuário do rio Tejipió. A análise cofenética foi significativa, com um $r>0,7$, apresentando três áreas com distintas influências marinha e fluvial.
\end{abstract}

$\overline{\text { Tropical Oceanography, Recife: v. 31, n. 2, p. 149-169, } 2003 .}$ 


\section{Abstract \\ Nutritional availability at Pina Basin and Tijipió River (Recife - PE - Brazil) in relation to nutrients and primary biomass (September/2000).}

This work was carried out at Pina Basin-PE in an estuary located at the internal area of the Port of Recife (in an urban zone formed by the confluence of the rivers Tejipió, Jiquiá, Jordão, Pina and Capibaribe). The objective was to assess the salinity dynamic and the conservative nutrient behavior, or non conservative nutrient behavior, in relation to the nutritional availability and the phytoplankton biomass development along the estuary. Water samples were collected at surface for hydrological and phytoplankton biomass data; and in all water column for salinity, using a Nansen bottle in 14 fixed stations at low-tide, in August/2000. Pina Basin is an estuary of salinity wedge, in its lower portion, homogeneous and partially mixed in the intermediate zone and at well mixed in the upper zone. The nutrients showed a non conservative behavior, with nitrite, nitrate and silicate being absorbed (mainly at low salinities) and ammonia and phosphate added to the ecosystem. The phosphate presented low variation showing that the buffer system occurs with this nutrient. The N:P relation varied from 14:1 to 3:1, showing that this environment is high dynamic in relation to nutritional availability. No nutrient limited although the values were lower than Redfield relation. Nitrogen was the main factor to influence the phytoplankton development, detected by the N:P relation and by the Main Component Analysis. Maximum suspended material was $60.0 \mathrm{mg} / \mathrm{l}$ and this is a high value, probably due to continental process in the studied area. This fact allows a better light penetration favoring the photosyntetic process in all stations. The environment is eutrophic with maximum chlorophyll of $105.20 \mathrm{mg} \cdot \mathrm{m}^{-3}$. In general, the biomass increased from the estuary mouth (station 1) to station 8, decreasing from this station until the rivers confluence and then increasing again in the stations at the Tijipió River. The cluster analysis presented three groups (diferent influences): one marine, one freshwater and third mixed group.

\section{INTRODUÇÃO}

Os ambientes estuarinos, dentre os ecossistemas aquáticos são altamente produtivos e ocupam importantes áreas da zona costeira. Os aspectos que explicam a razão de sua elevada produtividade são, o fluxo das marés, o aporte de nutrientes provenientes da drenagem terrestre e a existência de produtores primários, o que favorece a presença de elevadas densidades fitoplanctônicas.

Segundo Braga (1989), o estudo da dinâmica dos sais nutrientes serve como um bom indicador das relações abióticas e bióticas que ocorrem em águas marinhas tropicais. Estudos sobre produção primária e biomassa fitoplanctônica em regiões costeiras e oceânicas vem aumentando no país, uma vez que os conhecimentos sobre produção primária dão uma visão geral sobre a relação trófica e o fluxo de energia.

A Bacia do Pina está localizada na zona litorânea do Estado de Pernambuco e trata-se de uma área estuarina situada na parte interna do Porto do Recife, em plena zona urbana, sendo formada pela confluência dos rios Tejipió, Jiquiá, Jordão, Pina e pelo braço sul do Capibaribe. É um ambiente dinâmico do ponto de vista hidrográfico, característico do ecossistema estuarino. Recebe descargas de indústrias e efluentes domésticos e por ser uma área portuária, há um constante tráfego de embarcações. Sua extensão é de 3,6 km e largura variável, sendo a mínima de 0,26 km e a máxima de 0,86 km, perfazendo uma área total de aproximadamente 2,02 $\mathrm{km}^{2}$. Desempenha um papel de grande importância sócio-econômica, principalmente para a população circunvizinha de

Tropical Oceanography, Recife: v. 31, n. 2, p. 149-169, 2003. 
baixa-renda, que retiram o seu sustento da área, coletando diariamente peixes, moluscos e crustáceos (Feitosa, 1988).

Entre os estudos até então realizados na área, destacam-se: Coutinho (1961), Ottmann e Coutinho (1963) e Mabesoone e Coutinho (1970) que realizaram pesquisas sobre sedimentologia; Muniz e Mello (1976) sobre a ecologia e sistemática dos moluscos infaunais e Feitosa (1988), Koening et al. (1990), Silva-Cunha e Eskinazi-Leça (1990), Sant’Anna (1993) e Maia (1995) sobre a flora e a fauna planctônica.

Recentemente, Nascimento et al. (1999, 2000) desenvolveram estudos na Bacia do Pina, com o intuito de conhecer as variações sazonais e espaciais da biomassa, densidade e produtividade fitoplanctônica, associando-as aos parâmetros hidrológicos, assim como identificaram os principais grupos de algas planctônicas mais representativos na área estuarina, bem como, quantificaram a produtividade.

Este trabalho tem como objetivo analisar a dinâmica do estuário em relação a salinidade, determinar o comportamento conservativo ou não conservativo dos nutrientes ao longo do estuário, registrar a disponibilidade nutricional para o fitoplâncton, verificar a distribuição da biomassa fitoplanctônica ao longo do estuário na baixa-mar (período onde a influência continental é mais acentuada) e no final do período invernal.

\section{MATERIAL E MÉtodos}

Foram coletadas amostras de água na superfície para análises hidrológicas e determinação da biomassa fitoplanctônica em 14 estações na Bacia do Pina no final de setembro de 2000, na baixa-mar, utilizando-se garrafa de Nansen. A salinidade foi determinada em toda a coluna de água pelo método de titulação de Mohr-Knudsen descrito em Strickland e Parsons (1972) (Fig. 1).

Foram analisados: material em suspensão, de acordo com a técnica descrita por Melo et al. (1975); profundidade local, por uma ecossonda manual LCD Sounder Plastimo Echotest; temperatura, através de um termômetro comum de mercúrio; oxigênio dissolvido, pelo método de Winkler, descrito em Strickland e Parsons (1972); taxa de saturação do oxigênio, usando-se a tabela da Unesco (1973); transparência da água, medida com um disco de Secchi; pH, por intermédio de um pH-metro Beckman Zeromatic II; sais nutrientes (nitrito, nitrato fosfato) através das técnicas descritas em Strickland e Parsons (1972) e silicato por Grasshoff et al. (1983).

A biomassa foi determinada através do teor de clorofila $a$, utilizando-se o método espectrofotométrico da UNESCO (1966). Os cálculos foram realizados pelas equações de Parsons e Strickland (1963).

A filtração das amostras de clorofila realizou-se à vácuo com um filtro Schleicher \& Schüll de $0,45 \mu \mathrm{m}$ de porosidade e $0,47 \mathrm{~mm}$ de diâmetro.

\section{Tratamento dos dados}

Foram calculadas as similaridades entre os parâmetros hidrológicos e a biomassa fitoplanctônica, baseado no coeficiente de correlação momento-produto de Pearson. A matriz foi submetida a uma padronização (standardization) por fileiras, visando reduzir os efeitos das diferentes escalas. A classificação utilizada foi a aglomerativa hierárquica do "peso proporcional" (Weighted Pair Group Method Avarage Arithmetics - WPGMA).

Uma análise cofenética foi realizada, para medir a validade do agrupamento cujo valor $>0,7$ é significativo (Rohlf e Fisher, 1968). Todos estes cálculos foram feitos utilizando o programa computacional NTSYS (Numerical Taxonomy and Multivariate Analisys System) da Metagraphics Software Corporation, Califórnia - USA.

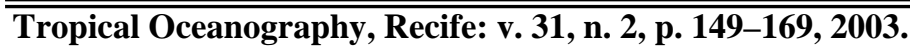


A análise dos componentes principais baseou-se na matriz de correlação momento-produto de Pearson, da qual foram extraídos o autovetor e o autovalor dos três principais componentes. Os cálculos foram feitos utilizando o programa computacional NTSYS (Numerical Taxonomy and Multivariate Analisys System) da Metagraphics Software Corporation, Califórnia USA.

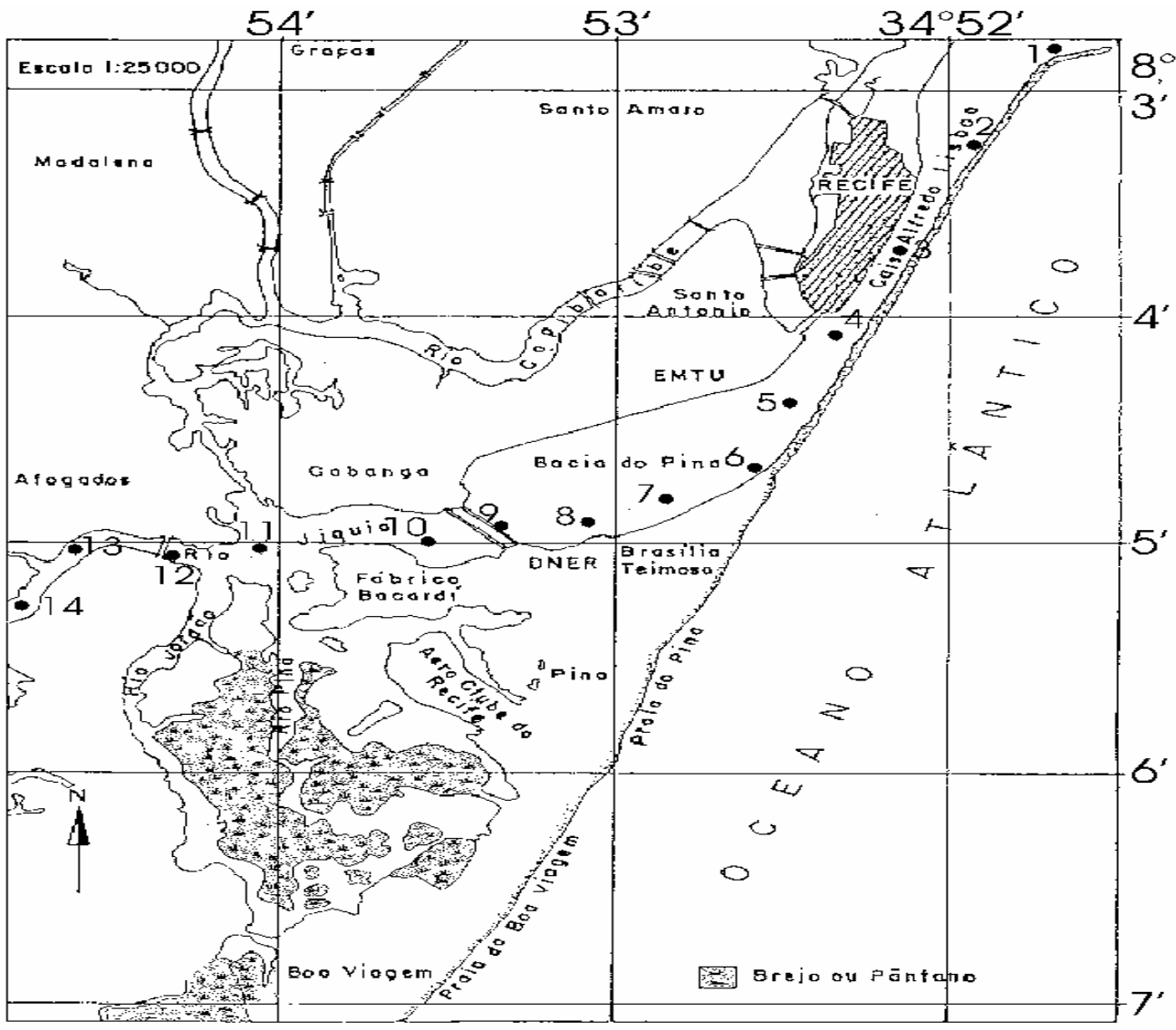

Figura 1 - Localização da área e estações de coleta

Estações: 1 - Boca da Barra; 2 - Bacia Portuária; 3 - Marco da cidade do Recife; 4 Desembocadura principal do Capibaribe; 5 - Antiga Mesbla Náutica; 6 - Iate Clube; 7 Entre o Iate e a antiga Companhia Aço Norte; 8 - Companhia Aço Norte; 9 - Entre as pontes que ligam Cabanga e o Pina; 10 - Em frente a Bacardi e a Compesa; 11 Confluência dos rios Tejipió e Jaboatão; 12 - Desembocadura do rio Tejipió; 13 - Rio Tejipió (após a ponte nova); 14 - Em frente ao viveiro de cultivo. 


\section{RESUltados E Discussão}

Ao longo de toda área estudada observou-se pequenas variações nos baixos valores encontrados para a transparência da água (Fig. 2 e Tab. 1). Os resultados obtidos a partir do disco de Secchi mostraram um mínimo de $0,30 \mathrm{~m}$ detectado nas estações 11 e 12, próximo à confluência dos rios Tejipió e Jordão, e máximo de 0,60m nas estações 2, 5 e 7 . O menor valor de transparência encontrado (estação 11) mostrou-se diretamente relacionado ao menor valor de clorofila $a$, em decorrência da alta turbidez da água, visto que a estação em questão encontra-se localizada na confluência dos rios que formam a bacia. Estando estes resultados de acordo com Feitosa (1988) que realizou estudos na área. Para outras áreas estuarinas os resultados mostraram-se semelhantes segundo Macedo et al. (1973), Macedo (1974), Passavante (1979) e Cavalcanti et al. (1981).

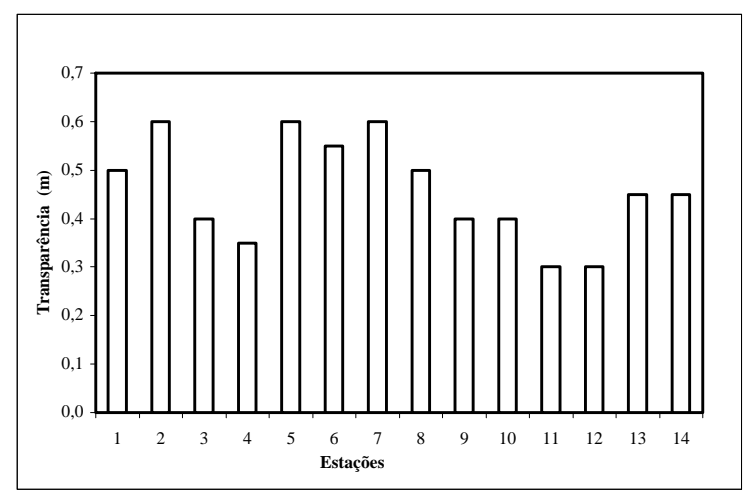

Figura 2 - Variação da transparência da água nas estações estudadas no mês de setembro de 2000.

A temperatura nas regiões tropicais apresenta uma grande estabilidade durante o ciclo sazonal, uma vez que depende grandemente do período e grau de insolação, variações meteorológicas, sendo estas condicionadas por períodos de maior ou menor nebulosidade (Flores Montes, 1996).

Tundisi (1970), verificou que as variações diurnas de temperatura são maiores em ambientes estuarinos que em águas costeiras e oceânicas, principalmente se o estuário é pouco profundo e grande parte do substrato fica exposto.

Na área em estudo, o comportamento térmico no mês de setembro é caracterizado por uma estabilidade horizontal da temperatura, sendo o valor máximo de $29^{\circ} \mathrm{C}$ nas estações 13 e 14 e o mínimo de $28^{\circ} \mathrm{C}$ nas estações de 1 a 8 (Tab. 1 ).

Considerando os resultados obtidos referente a saturação do oxigênio, ocorreram algumas oscilações, estando os valores na sua maioria abaixo de $50 \%$, com concentrações de $\mathrm{O}_{2}$ próximas à 1ml/l. Com exceção da estação 1, que apresentou um valor de 4,96 ml/l (96,70\% de saturação), as demais apresentaram déficts de oxigênio, demostrando comprometimento da área em relação a este parâmetro. Os dados estão coerentes com os resultados obtidos por Feitosa (1988) para o mesmo local de estudo. Este autor obteve índices de saturação que variaram desde zona supersaturada até zona poluída, incluindo nesse estudo tanto a influência da baixa-mar quanto da preamar.

Segundo Chamixaes (1984), os baixos teores de saturação do $\mathrm{O}_{2}$ comprometem seriamente todos os níveis tróficos do ecossistema. Esse estado é decorrente, principalmente, da intensa

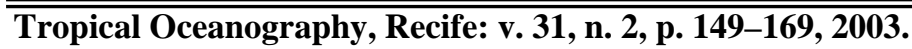


poluição orgânica na Bacia do Pina. Em relação ao percentual de saturação foi registrado mínimos de $18,91 \%$ (2,50 ml/l) e 18,27\% (2,90 ml/l) nas estações 6 e 7, respectivamente (Fig. 3 e Tab. 1).

Para o estuário do rio Jaguaribe, Santos-Fernandes (1997), observou no regime da baixamar, que na maioria das ocasiões as taxas de saturação de $\mathrm{O}_{2}$ foram superiores a $50 \%$, sugerindo ser este um ambiente influenciado pelas características marinhas devido a sua pequena extensão e ao grande volume de água costeira que penetra no estuário. Na Bacia do Pina o fluxo marinho que entra pela estação 1 influencia toda a área, principalmente na preamar, porém o presente trabalho foi realizado na baixa-mar e no final de um período invernal com alta intensidade pluviométrica, quando a drenagem terrestre é alta.

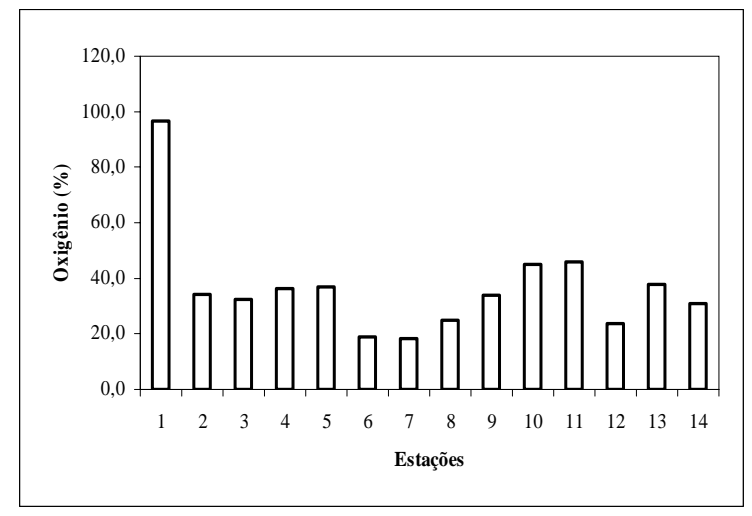

Figura 3 - Variação da saturação do Oxigênio nas estações estudadas.

De um modo geral, as águas da Bacia do Pina apresentaram-se alcalinas, com $\mathrm{pH}$ variando entre 7,91 e 8,76 sendo registrado o menor valor para a estação 1 e o maior para a estação 10 (Tab. 1). Os valores mantiveram-se dentro dos limites efetivos para a vida marinha que, segundo Perkins (1977), encontram-se compreendidos entre 6,5 e 9,0.

Feitosa (1988), na Bacia do Pina, verificou estratificação salina mais evidente na preamar com maiores valores na camada profunda. O autor cita que a estação com maior profundidade teve uma estratificação mais evidenciada, demonstrando que o regime de salinidade e o tipo de estuário mudam de acordo com a maré, com a intensidade pluviométrica, e com a vazão dos rios.

Na boca da barra (estação 1), a Bacia do Pina pode ser caracterizada como um estuário de cunha salina (Fig. 4 e Tab. 2). Nas duas estações seguintes, se observa uma homogeneidade tanto horizontal como vertical, mantendo-se a salinidade ao redor de 18. A partir da quarta até a sexta estação, as isoalinas estão mais inclinadas, indicando uma mistura vertical parcial. Em seguida o estuário se torna totalmente misturado verticalmente depois da desemborcadura do rio Capibaribe (próximo à estação quatro) até a confluência dos rios Tejipió e Jordão, com um gradiente horizontal descendente. Este caso indica que a água se move para cima e para baixo com mais facilidade, gerando turbulência, criando uma área de mistura maior que no início da bacia. Esta estrutura de salinidade está claramente influenciada pela morfologia de fundo, que forma uma barreira na estação 5, impedindo que o fluxo da água salina em forma de cunha continue entrando na Bacia e, ao mesmo tempo, mantendo-a represada nas proximidades da estação 4 (área de maior profundidade).

A principal fonte de nutrientes para os estuários são as descargas fluviais (Travassos, 1991; Hansen et al., 1995; Herrera-Silveira e Comin, 1995; Prego, 1994); águas da drenagem terrestre

Tropical Oceanography, Recife: v. 31, n. 2, p. 149-169, 2003. 
provocada pelas chuvas (Flores Montes, 1996); ressuspensão de nutrientes depositados ou regenerados no sedimento (Chester, 1990); águas costeiras marinhas (Boynton et al., 1982; Fan e Jin, 1989; Millero e Sohn, 1992); e mais recentemente, aportes de nutrientes antropogênicos, como a precipitação aérea, causada por sólidos em suspensão na atmosfera devido à poluição, provocando eutrofização em diversas regiões costeiras e aumentado a produção primária nova (Paerl, 1995; Rasmussen, 1995; Tett e Walne, 1995).

Tett e Walne (1995), relataram que o enriquecimento das águas por nutrientes, provocando um acelerado crescimento de algas, pode produzir um desequilíbrio indesejável na comunidade planctônica e na qualidade da água.

\section{Estações}

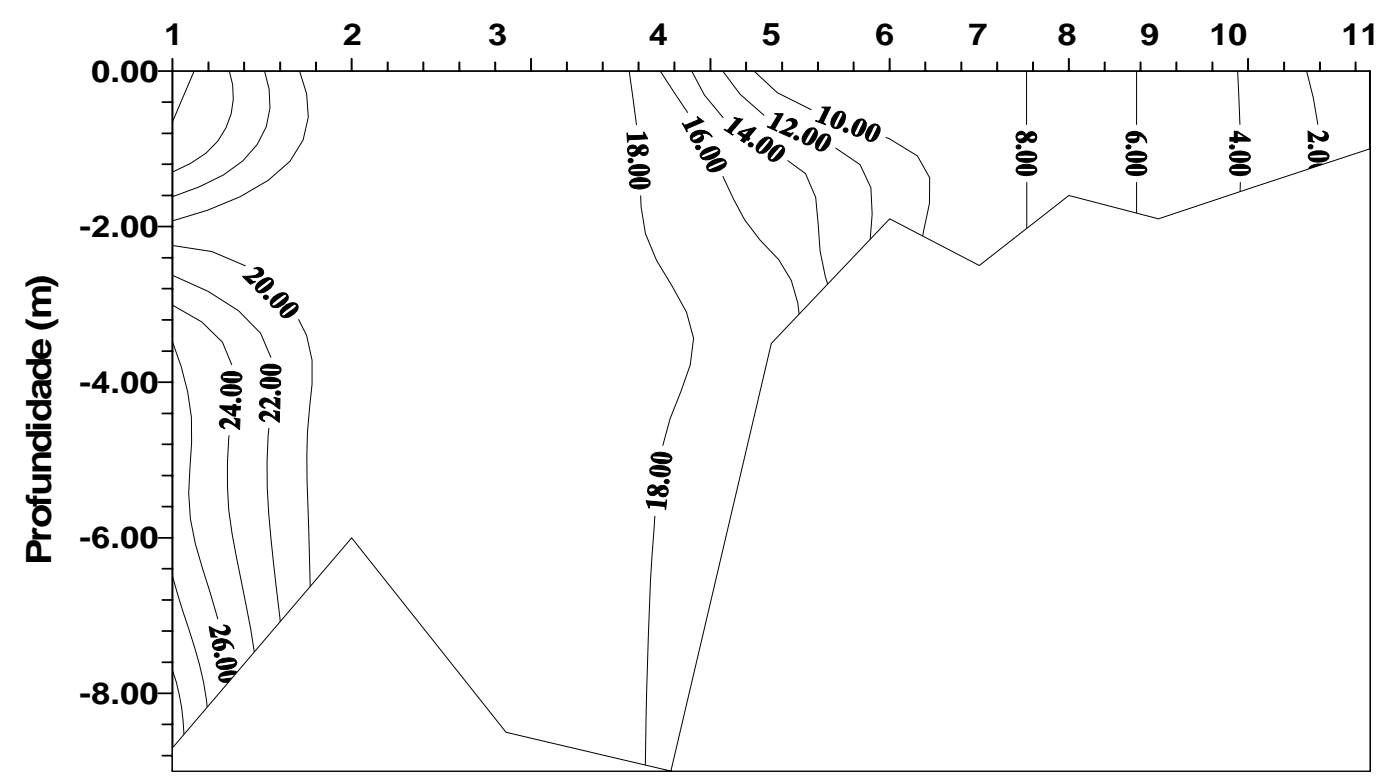

Figura 4 - Distribuição vertical da salinidade nas estações estudadas.

Aidar et al. (1993) em Ubatuba (SP) observaram que o nitrogênio, o fósforo e a sílica, comportam-se de forma não conservativa na dependência direta de processos de demanda biológica pela biomassa fitoplanctônica na zona eufótica e dos aportes devido a processos físicos (advecção, intrusão de águas mais ricas), biológicos (regeneração), climáticos (chuvas) e drenagem continental.

Feitosa (1988) verificou que índices elevados de sais nutrientes podem ser provenientes dos sedimentos de fundo e da drenagem do solo através dos diversos rios que atravessam a Bacia do Pina. 


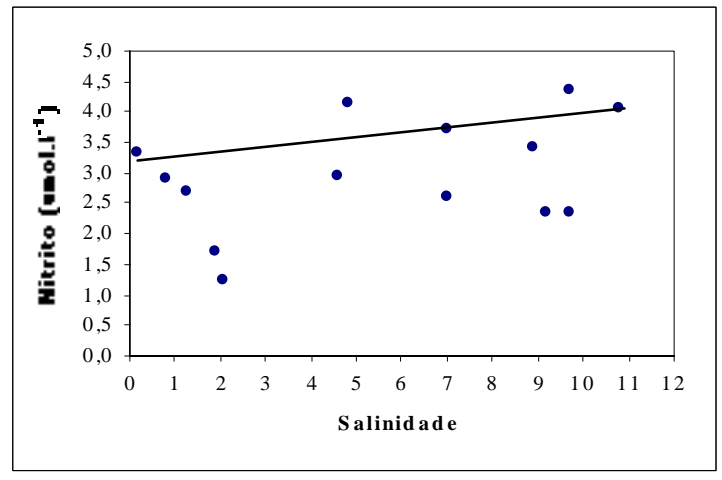

Figura 5 - Diagrama de mistura do Nitrito de águas superficiais nas estações analisadas.

No presente estudo o nitrito apresentou concentrações elevadas $\left(1,26\right.$ - 4,36 $\left.\mu \mathrm{mol}^{-1}{ }^{-1}\right)$ (Fig. 5 e Tab. 1). Este nutriente, sendo um produto intermediário e instável das reações de denitrificação e de nitrificação, é comum que as suas concentrações na água sejam, em geral, relativamente mais baixas do que as das outras formas de nitrogênio dissolvido, como as dos íons de amônia $\left(\mathrm{NH}_{4}{ }^{+}\right)$e de nitrato $\left(\mathrm{NO}_{3}{ }^{-}\right)$, o que tem sido demonstrado em inúmeras pesquisas e corroborando, no caso das águas estuarinas por Burton (1976).

No que se refere ao nitrato, a tabela 1 mostra uma maior concentração na estação 4 $\left(29,77 \mu \mathrm{mol} . \mathrm{l}^{-1}\right)$ e menor na estação $13\left(1,74 \mu \mathrm{mol}^{-1} \mathrm{l}^{-1}\right)$. Este composto apresentou um comportamento não conservativo, observando-se em quase toda a bacia remoção do mesmo, principalmente nas baixas salinidades (Fig. 6).

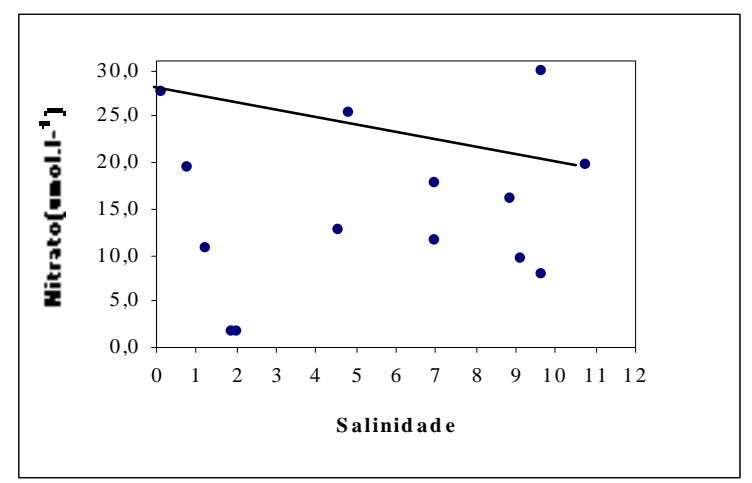

Figura 6 - Diagrama de mistura do Nitrato de águas superficiais nas estações analisadas.

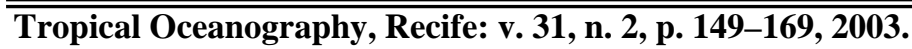


A figura 7 indica que a amônia está sendo adicionada em grande parte ao ambiente com concentrações características de um ambiente eutrófico. O maior valor foi registrado na estação 14 $\left(16,18 \mu \mathrm{mol}^{-1} \mathrm{l}^{-1}\right)$, última do rio Tejipió. Apesar da descarga dos rios ter a tendência de aumentar fortemente as concentrações de amônia, isto não ocorreu na estação 11 (menor concentração $3,99 \mu \mathrm{mol} \mathrm{l}^{-1}$ ), o que pode indicar que nesta localização estaria ocorrendo uma oxidação mais rápida, favorecido por uma maior oxigenação da água, provocada pela turbulência que ocorre neste ponto devido à confluência dos rios.

Madden et al. (1988) em estudo em Fourleagne Bay - USA, observaram que a variabilidade da amônia em relação a salinidade não foi conservativa. Os autores relatam que isso indica uma forte mediação biológica, aumentando a concentração na coluna de água através de processos de regeneração e/ou ressuspensão, o que influenciaria a concentração de amônia no estuário.

Catalano (1987), considera a amônia como um nutriente limitante para o crescimento do fitoplâncton, não sendo uma forma estável do nitrogênio. Porém, no presente trabalho, os processos de adição aparentemente superam os de remoção (Fig. 7).

Os elevados valores detectados de nitrito e amônia, demonstram que o processo de nitrificação é um dos mais relevantes no ambiente enfocado. O aumento do nutriente nitrogenado é proveniente dos despejos orgânicos domésticos, porém não se deve descartar também o aporte a partir dos sedimentos do fundo, uma vez que a área em frente ao porto do Recife é consideravelmente dinâmica do ponto de vista físico, tanto em função dos movimentos da massa d’água como também da interferência humana no revolvimento deste sedimento através do processo de dragagem, comumente realizado na área do complexo portuário (Ressurreição, 1990).

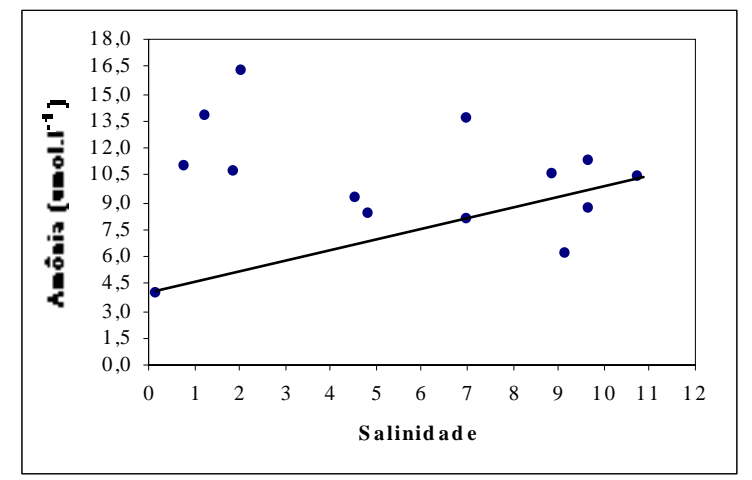

Figura 7 - Diagrama de mistura da Amônia de águas superficiais nas estações analisadas.

No que diz respeito ao fosfato, verifica-se que as concentrações variaram de modo bastante irregular em relação às estações, sendo registrada na estação $12\left(6,79 \mu \mathrm{mol}^{-1} \mathrm{I}^{-1}\right)$ a maior concentração e na estação $2\left(2,86 \mu \mathrm{mol}^{-1} \mathrm{l}^{-1}\right)$ a menor. Este nutriente apresentou um comportamento não conservativo, com processos de adição mais acentuados que os de remoção ao longo da bacia (Fig. 8).

Maglioca (1971), relatou que as águas dos rios Amazonas e Pará transportam grandes quantidades de material em suspensão prontos a fornecer fósforo ao atingir o estuário e que pela sedimentação e conseqüente redução da turbidez, o fósforo liberado é prontamente consumido. Ao longo da bacia se observou que o processo de adição é mais evidente e as concentrações de fosfato

$\overline{\text { Tropical Oceanography, Recife: v. 31, n. 2, p. 149-169, } 2003 .}$ 
não apresentaram variações tão acentuadas como as do nutriente nitrogenado (Figs. 6 e 8 e Tab. 1). Este equilíbrio entre os processos de adição e absorção está relacionado ao efeito tampão que ocorre em áreas estuarinas através da dessociação e adsorção do fósforo por partículas em suspensão.

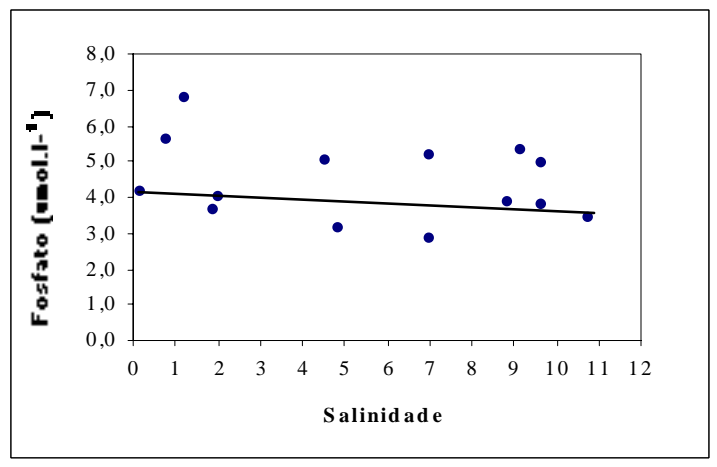

Figura 8 - Diagrama de mistura do Fosfato de águas superficiais nas estações analisadas.

Os valores observados na relação N/P para as estações ao longo da bacia, variaram entre 14:1 (estações 2 e 4) e 3:1 (estação 7). Observando a figura 9, verifica-se uma tendência desta relação em diminuir da estação 1 à estação 14. Neste estudo específico não se pode dizer que o nitrogênio seja um elemento limitante $(\mathrm{N}: \mathrm{P}<16: 1)$, devido à concentração elevada deste nutriente e por ser uma área estuarina de grandes flutuações em relação à taxa de Redfield. Se observou, porém, que a disponibilidade de nitrogênio é menor nas estações com menor influência marinha. Isto pode ser comprovado pela figura 6, onde se observa um maior consumo em salinidades baixas, coincidindo com valores altos de clorofila $a$ (Tab. 1).

É comum a alternância entre o fósforo e o nitrogênio como limitantes da produção na presença de um gradiente salino, sendo o fósforo limitante nas águas menos salinas, sob maior

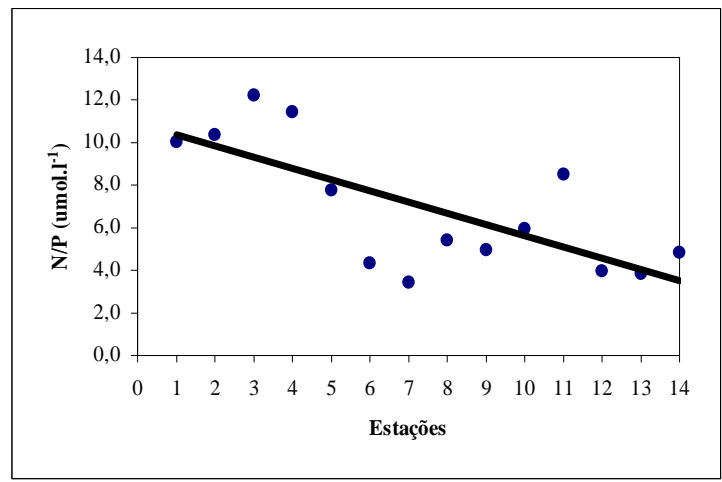

Figura 9 - Relação N:P das amostras superficiais nas estações analisadas. 
influência terrígena, e o nitrogênio nas águas com maior salinidade, sob maior influência marinha (Graneli e Graneli, 1982; Graneli, 1987; Caraco et al., 1987). Na Bacia do Pina, ocorreu comportamento oposto, sendo os maiores valores de N/P registrados nas estações mais próximas à boca da barra (estações 1 à 5).

O silicato apresenta também um comportamento não conservativo, sendo bastante expressiva sua remoção no ambiente, principalmente entre as salinidades de 2,00 a 9,00 (Fig. 10). A concentração desse nutriente variou de $156,84 \mu \mathrm{mol}^{-l^{-1}}$ na estação 2 à $476,04 \mu \mathrm{mol} . \mathrm{l}^{-1}$ nas estações 10, 11 e 12 (Tab. 1).

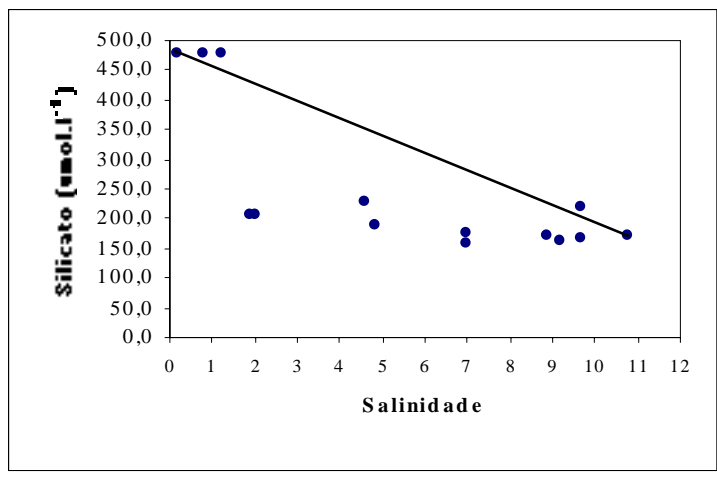

Figura 10 - Diagrama de mistura do Silicato de
águas superficiais nas estações
analisadas.

A distribuição de silicato em águas costeiras é geralmente maior do que no oceano aberto devido a descarga dos rios (Millero, 1996), o que foi constatado no presente trabalho. Dentre os sais nutrientes dissolvidos, o silicato foi o que apresentou os valores mais elevados, seguido pelo nitrato, fosfato e nitrito, fato semelhante ao encontrado por Feitosa (1988) na Bacia do Pina.

A importância das concentrações de sílica no meio, está intimamente relacionada à ecologia das diatomáceas, cuja frústula de sílica é a base estrutural da parede celular (Darley, 1982). Por esta razão, a disponibilidade de sílica na água é de vital importância para as diatomáceas, pois dela dependem os processos de reprodução, crescimento e produção destes componentes do fitoplâncton, influenciando diretamente na distribuição das mesmas nos corpos de água naturais (Moreira, 1994).

Nascimento et al. (2000), trabalhando durante os anos de 97/98 na Bacia do Pina, encontraram as seguintes variações de nutrientes: silicato de 7,58 a $86,46 \mu \mathrm{mol}^{-1} \mathrm{l}^{-1}$ fosfato de 0,37 a $4,15 \mu \mathrm{mol} . \mathrm{l}^{-1}$ e nitrato de 0,55 a $16,32 \mu \mathrm{mol}^{-l^{-1}}$. Valores bem mais baixos que os encontrados no presente trabalho. Nestes dois anos (97/98), o inverno foi atípico, ou seja, devido às secas que ocorreram no nordeste as chuvas foram escassas, diminuindo portanto o fluxo fluvial para a Bacia do Pina.

Analisando o material em suspensão, o valor mínimo foi de $20,0 \mathrm{mgl}^{-1}$ detectado nas estações 2 e 12, e o maior obtido foi de $60,0 \mathrm{mgl}^{-1}$ na estação 11, localizada na confluência dos rios, ponto de maior acúmulo de material em suspensão (Fig. 11 e Tab. 1). Esses teores foram relativamente baixos, levando-se em consideração a área estudada. Porém o processo de

Tropical Oceanography, Recife: v. 31, n. 2, p. 149-169, 2003. 
sedimentação na maré baixa, contribui para o acumulo do material em suspensão nas camadas mais profundas e no sedimento, já que as coletas só foram realizadas na superfície.

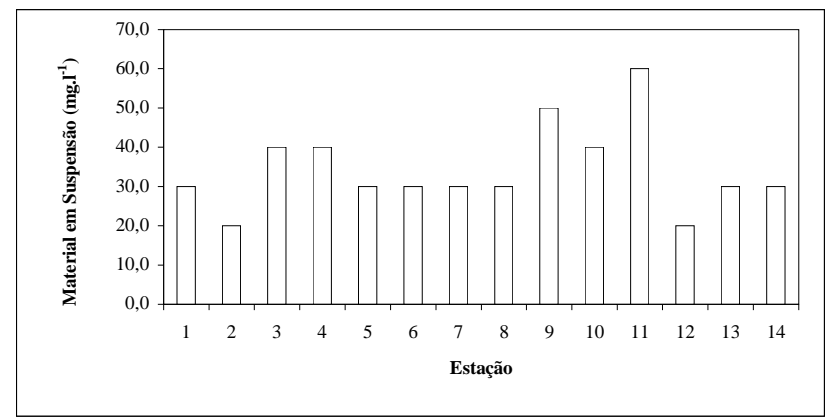

Figura 11 - Material em suspensão total nas estações analisadas.

Em relação à clorofila $a$, esta apresentou-se mais baixa na estação $11\left(25,65 \mathrm{mg} / \mathrm{m}^{3}\right)$, seguida pela estação 1 e 3 (Tab. 1). De um modo geral a biomassa aumentou da boca da barra (estação 1) até a estação 8, diminuindo a partir daí até a estação 11 (ponto de confluência dos rios) e elevando-se novamente nas estações 12, 13 e 14 (máxima de 105,20 mg/m³), estações estas localizadas no rio Tejipió (Fig. 12). Feitosa (1988), constatou que as concentrações mais elevadas de clorofila $a$ ocorreram durante a baixa-mar na Bacia do Pina no verão, com um valor máximo de $360 \mathrm{mg} / \mathrm{m}^{3}$ e em setembro na baixa-mar na superfície, alcançou um valor de $193,64 \mathrm{mg} / \mathrm{m}^{3}$, concentração mais elevada que a encontrada no presente trabalho. Estas intensas variações estão associadas ao clima, ou seja, invernos pouco chuvosos ou com intensidade pluviométrica acentuada.

Costa (1991), comentou que a variação da clorofila $a$ é um reflexo da distribuição quantitativa do fitoplancton sendo influenciada pelas características nutricionais das diferentes massas d'água. Na Bacia do Pina os elevados teores de clorofila $a$ total foram registrados nas estações mais influenciadas por águas fluviais, fato que permite considerar o ecossistema como sendo um ambiente eutrófico, mesmo ocorrendo oscilações das concentrações em relação às estações. Ressureição (1990), detectou, para a região do Porto do Recife, uma concentração no teor de pigmentos fotossintetizantes decrescendo à medida que se afasta da costa.

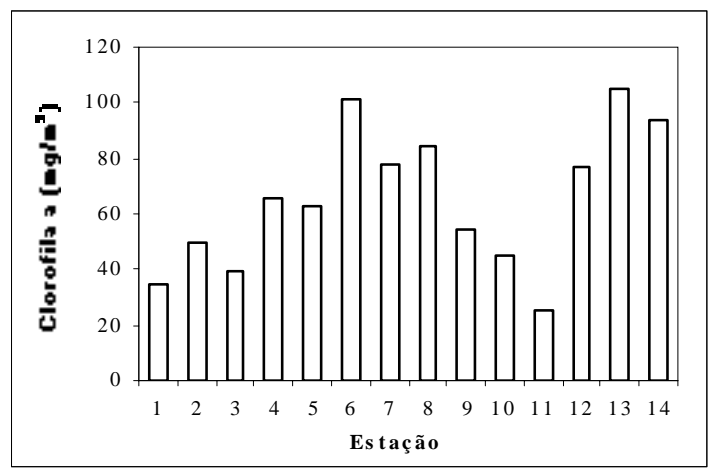

Figura 12 - Distribuição superficial da Clorofila $a\left(\mathrm{mg} / \mathrm{m}^{3}\right)$ nas estações analisadas. 
Relacionando os valores de clorofila $a$ com a disponibilidade nutricional N:P, se observa que as maiores concentrações coincidiram com os menores valores de N:P. Já que a transparência da água e o material em suspensão não variaram significativamente, deve-se concluir que o $\mathrm{N}$ é o elemento que mais influenciou no desenvolvimento fitoplanctônico.

A análise cofenética foi significativa, apresentando um $r>0,7$. Evidenciaram-se dentro desse único grupo de amostras, três subgrupos (Fig. 13): marinho.

Subgrupo 1: Constituído pelas estações 1, 2, 3, 4 e 5 as quais apresentaram maior fluxo

Subgrupo 2: Constituído pelas estações 6, 7, 8, 12, 13 e 14 que apresentaram fluxo intermediário, com características marinhas e limnéticas.

Subgrupo 3: Caracterizou-se pelas estações 9, 10 e 11 que apresentaram um maior fluxo limnético, por se localizarem em área onde é maior a influência dos rios que formam a Bacia.

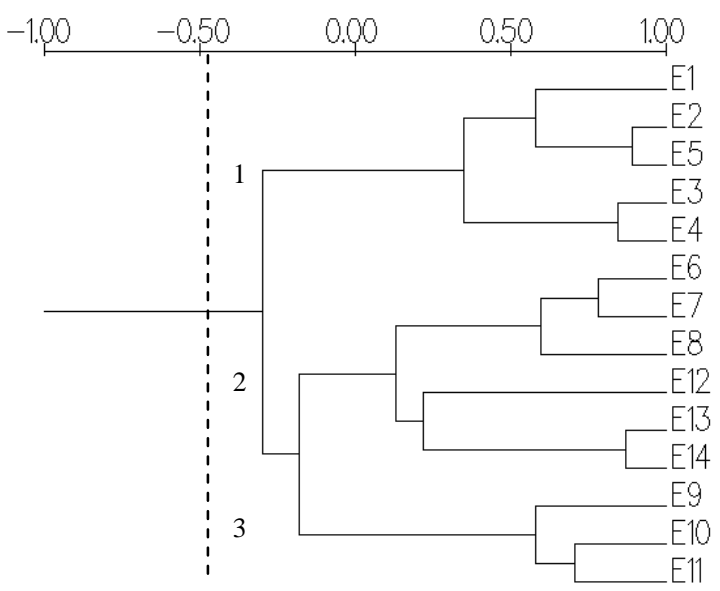

Figura 13 - Dendrograma da associação das estações, na Bacia do Pina.

A análise dos Componentes Principais pode ser vista na Tabela 3. Os três primeiros fatores explicam 80,08\% das variações ocorridas no sistema estuarino da Bacia do Pina.

O fator 1 explicou 36,40\% da variação dos dados e associou diretamente a temperatura, $\mathrm{pH}$, silicato e estes correlacionados inversamente com a salinidade e a transparência, indicando desta maneira uma forte influência marina quanto a variação de alguns parâmetros estudados, apesar das coletas terem sido realizadas na baixa-mar.

O fator 2 explicou 33,24\% da variação dos dados e correlacionou diretamente nitrito, nitrato e material em suspensão e estes inversamente correlacionados com a amônia e clorofila, indicando uma maior influência limnética principalmente nos valores de clorofila $a$. Neste caso, o nitrogênio é o fator mais importante que está acelerando ou diminuindo o desenvolvimento fitoplanctônico na Bacia do Pina, sendo o nitrato o composto mais assimilado. Fato este comprovado pela relação N:P ao longo do estuário (Fig. 9). A amônia, sendo uma fase intermediária do ciclo do nitrogênio, torna-se importante como fonte de nitrato para a área, já que ele está sendo mais adicionado que absorvido (Fig. 7).

$\overline{\text { Tropical Oceanography, Recife: v. 31, n. 2, p. 149-169, } 2003 .}$ 
O fator 3 explicou apenas 10,44\% da variação dos dados estando apenas o fosfato neste fator, indicando não ser limitante neste ecossistema.

\section{CONCLUSÕES}

1. Do ponto de vista da salinidade, a Bacia do Pina apresentou-se como um estuário de cunha salina nas estações mais externas, homogêneo nas intermediárias e parcialmente misturado nas estações mais internas.

2. Os nutrientes apresentaram um comportamento não conservativo, sendo o nitrito, nitrato e o silicato os mais absorvidos (principalmente em salinidades mais baixas), e amônia e o fosfato adicionados ao ecossistema via atividades antropogênicas, sendo que as pequenas variações do fosfato ao longo do trecho, indicam a existência de um sistema tampão regulando este nutriente.

3. A relação N:P, apresentou-se sempre abaixo da relação de Redfield, não comprometendo a produtividade do estuário. $\mathrm{O}$ nitrogênio foi $\mathrm{o}$ fator principal que influenciou $\mathrm{o}$ desenvolvimento fitoplanctônico, indicado pelos valores $\mathrm{N}: \mathrm{P}$ e pela Análise dos Componentes Principais.

4. A área se apresentou eutrófica, devido à quantidade acentuada de clorofila $a$ com valores acima de $30 \mathrm{mg} / \mathrm{m}^{3}$

5. A análise cofenética foi significativa, apresentando um $r>0,7$. Evidenciaram-se dentro desse único grupo de amostras, três subgrupos significativos ou áreas bem delimitadas com influências marinha e/ou limnética.

\section{REFERÊNCIAS BIBLIOGRÁFICAS}

AIDAR, E.; GAETA, S. A.; GIANESELLA - GALVÃO, S. M. F.; KUTNER, M. B. B.; TEIXEIRA, C. Ecossistema Costeira Subtropical: nutrientes dissolvidos, fitoplâncton e clorofila- $a$ e suas relações com as condições oceanográficas na região de Ubatuba, SP. Bol. Inst. Oceanogr. de São Paulo, São Paulo, v. 10, p. 9- 43, 1993.

BOYNTON, W. R.; KEMP, W. M.; KEEFE, C. W. A. Comparative analysis of nutrients and other factors influencing estuarine phytoplankton production. In: KENEDDY, V. S. (Ed), Estuarine Comparisons, London: Academic Press, p. 69- 90, 1982.

BRAGA, E. S. Estudo dos nutrientes dissolvidos nas águas da enseada das Palmas, Ilha Anchieta (Ubatuba, SP), com ênfase às formas nitrogenadas e contribuição por aportes terrestres e atmosféricos. São Paulo, 1989. 207 f. Dissertação (Mestrado em Oceanografia) - Inst. Oceanogr. de São Paulo, USP.

BURTON, J. D. Basic properties and processes in estuarine chemistry. In: BURTON, J. D.; LISS, P. S. (Eds), Estuarine Chemistry, London: Academic Press, p. 1-31, 1976.

CARACO, N.; TAMSE, A.; BOUTROS, O.; VALIELA, I. Nutrient limitation of phytoplankton growth in brackis in coastal ponds. Canadian J. of Fish. Aguat. Sci., v. 44, p. 473-476, 1987.

$\overline{\text { Tropical Oceanography, Recife: v. 31, n. 2, p. 149-169, } 2003 .}$ 
CATALANO, G. An improved method for the determination of ammonia in seawater. Marine Chemistry, Amsterdam, v. 20, n. 3, p. 289-295, jan. 1987.

CAVALCANTI, L. B.; MACEDO, S. J. de.; PASSAVANTE, J. Z. de O. Estudo ecológico da região de Itamaracá, Pernambuco - Brasil. XXI. Caracterização do Canal de Santa Cruz (PE) em função dos parâmetros físico - químicos e pigmentos fotossintéticos. Trab. Oceanogr. da Univ. Fed. PE. Recife, v. 16, p. 157-216, 1981.

CHAMIXAES, C. B. C. B. Produção primária do fitoplâncton relacionada com as condições ecológicas do Açude de Apipucos. 1984. 242 f. Dissertação (Mestrado em Criptógamos) CCB, UFPE, Recife.

CHESTER, R. Marine Geochemistry. Londres: Chapman e Hall, 1990. 698 f.

COSTA, K. M. P. Hidrobiologia e Biomassa Primária da Região Nordeste do Brasil entre as

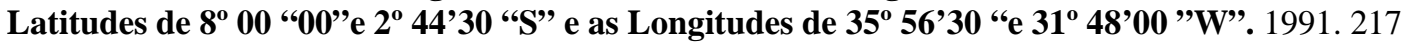
f. Dissertação (Mestrado em Oceanografia) CTG, UFPE, Recife.

COUTINHO, P. A. Estudo das Condições de Sedimentação do Porto do Recife. 1961. 29 f. Relatório de graduação - escola geológica da Universidade do Recife. (manuscript). Recife.

DARLEY, W. M. Algal biology: a physiological approach. Oxford: Blackwell Scientific Publications, 1982. 168 f. (Basic microbiology).

FAN, A.; JIN, X. Tidal effect on nutrient exchange in xiangshan Bay, China. Marine Chemistry, Amsterdam, v. 27, n. 3-4, p. 254-281, Oct. 1989.

FEITOSA, F. A. do N. Produção primária do fitoplancton relacionada com parâmetros bióticos e abióticos da Bacia do Pina (Recife - Pernambuco, Brasil). 1988. 220 f. Dissertação (Mestrado em Oceanografia), CTG, UFPE, Recife.

FLORES MONTES, M. Variação nictmeral do fitoplâncton e parâmetros hidrológicos no Canal de Santa Cruz (Itamaracá, PE). 1996. 119 f. Dissertação (Mestrado em Oceanografia), CTG, UFPE, Recife.

GRANELI, E. Nutrient Limitation of phytoplankton biomass in a brackish water bay highly influenced by river discharge. Estuar. Cost. Shelf. Sci., London, v. 25, p. 555-565, 1987.

GRANELI, E.; GRANELI, W. Entrophication and dinoflagellate blooms in swedish coastal waters - possible causes and cauntermeasures. In: International symposium on utilization of coastal ecosystems: Planning, Pollution and Productivity, v. 5, n. 2, 1982, Rio Grande. Resumos... Rio Grande: FURG, 1982, p. 61.

GRASSHOFF, K.; EHRHARDT, M.; KREMLING, K. Methods of Seawater Analysis. 2. ed. Florida: Verlage Chemie, 1983. $417 \mathrm{f}$.

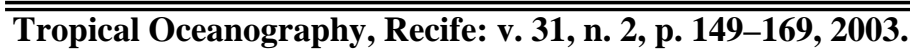


HANSEN, I. S.; AERTBJERG, G.; RICHARDSON, K. A scenario analysis of effects of reduced nitrogen input on oxygen conditions in the Kattegat and the Belt Sea. Ophelia, Heldinggor, v. 42, p. 75-93, Sept. 1995.

HERRERA -SILVEIRA, J. A.; COMIN, F. A. Nutrient fluxes in a tropical coastal lagoon. Ophelia, Heldinger, v. 42, p. 127-146, Sept. 1995.

KOENING, M. L.; ESKINAZI - LEÇA, E.; FEITOSA, F. A. N. Biomassa fitoplanctônica da Bacia do Pina. In: REUNIÃO NORDESTINA DE BOTÂNICA, 14., 1990, Resumos... Recife: SUDENE, 1990.

MABESOONE, J. M.; COUTINHO, P. N. Litorral and Shallow marine geology of Northeastern Brazil. Trab. Oceanogr. da Univ. Fed. de PE, Recife, v. 12, p. 1-214, 1970.

MACEDO, S. J. Fisioecologia de alguns estuários do Canal de Santa Cruz (Itamaracá Pernambuco). 1974. 121 f. Dissertação (mestrado), Universidade de São Paulo, São Paulo.

MACEDO, S. J. de; LIRA, M. E. F.; SILVA, J. E. da. Condições hidrológicas do Canal de Santa Cruz, Itamaracá, PE. B. Rec. Nat., SUDENE, Recife, v. 11, n. 1/2, p. 55-92, 1973.

MADDEN, C. J.; DAY, J.W.; RANDALL, J. M. Freshwater and marine coupling in estuaries of the Mississipi River deltaic laih. Liminol. Oceanogr. Lawrenu, v. 33, n. 4, part II, p. 98-104, july. 1988.

MAGLIOCCA, A. Some chemical aspects off the Amazon and Pará Rivers, Brazil. Bol. Inst. Oceanogr. de São Paulo, São Paulo, v. 20, p. 61-84, 1971.

MAIA, P. R. Composição e densidade fitoplanctônica da Bacia do Pina (Recife, Pernambuco). 1995. 135 f. Dissertação (Mestrado em Oceanografia), CTG, UFPE, Recife.

MELO, U.; SUMMERHAYES, C. P.; TONER, L. G. Metodologia para o estudo do material em suspensão na água do mar. Bol. Téc. Petrobrás, Rio de Janeiro, v. 18, n. 3/4, p. 115-127, 1975.

MILLERO, F. J. Chemical Oceanography. 2 ed. New York: CRC Press, 1996. $531 \mathrm{f}$.

MILLERO, F. J., SOHN, M. L. Chemical Oceanography. Boca Raton: CRC Press, 1992. 531 f.

MOREIRA, M. O. P. Produção do Fitoplâncton em um Ecossistema Estuarino Tropical (estuário do Rio Cocó, Fortaleza, Ceará). 1994. 338 f. Dissertação (Mestrado em Oceanografia), CTG, UFPE, Recife.

MUNIZ, G. C. B.; MELLO, R. L. S. Moluscos bivalves infaunais da região estuarina da Bacia do Pina, Recife, Pernambuco, Brasil. Anais da Univ. Fed. Rural de PE, Recife, v. 3, p. 205 - 219. 1976.

NASCIMENTO, F. C. R. do; FEITOSA, F. A. do N.; MUNIZ, K. Variação espacial e temporal da produção fitoplanctônica fracionada na Bacia do Pina relacionada com parâmetros hidrológicos (Recife - Pernambuco - Brasil). In: SEMANA NACIONAL DE OCEANOGRAFIA, 12, Rio de Janeiro. Resumos... Rio de Janeiro: Universidade Estadual do Rio de Janeiro, 1999. p. 307-309.

$\overline{\text { Tropical Oceanography, Recife: v. 31, n. 2, p. 149-169, } 2003 .}$ 
NASCIMENTO, F. C. R. do; FEITOSA, F. A. do N.; MUNIZ, K. Avaliação do padrão sazonal e espacial da densidade fitoplanctônica e parâmetros ambientais na Bacia do Pina - PE - Brasil. In:, SEMANA NACIONAL DE OCEANOGRAFIA, 13, Itajaí -Santa Catarina. Anais... Santa Catarina: Universidade do Vale do Itajaí. Centro de Ciências Tecnológicas da Terra e do Mar, p.475-477. 2000.

OTTMANN, F.; COUTINHO, P. N. Estudes sédimentologiques dans le port de Recife (Brésil). Cashiers Oceanographiques, v. 15, p. 161-169, 1963.

PAERL, H. W. Coastal eutrophication in relation to atmosphera nitrogen deposition: current perspectives, Ophelia, Helding, v. 41, p. 237-259, Feb. 1995.

PARSONS, T. R.; STRICKLAND, J. D. H. Discussion of spectrophotometric determination of marine plankton pigments, with revised equations of as certaninig clorophyll a and caratenoids. Journal of Marine Research, New Haven, v. 21, n. 3, p. 155-163. 1963.

PASSAVANTE, J. Z. de O. Produção primária do fitoplâncton do Canal de Santa Cruz (Itamaracá - PE). 1979. 188 f. Tese (Doutorado em Oceanografia), Instituto Oceanográfico da Universidade de São Paulo, São Paulo.

PERKINS, E. J. Inorganic Wastes. In: Lenihan, J., Fletcher, w. w. (eds.) The marine environment. Glasgow: Blackie \& Son limited, 1977. p. 70-101.

PREGO, R. Nitrogen interchanges generated by biogeochemical processes in a galician. Marine Chemistry, Amsterdam, v. 45, n. 1/2, p. 167-176, jan. 1994.

RASMUSSEN, B. Stratification and wind mixing in the southern kattegat, Ophelia, Helding, v. 42, p. 313-334, Sep. 1995.

RESSURREIÇÃO, M. G. Variação anual da biomassa fitoplanctônica da Plataforma Continental de Pernambuco: perfil em frente ao Porto do Recife (08 $03^{\circ} 38^{\prime \prime}$ lat. S; 34²'28”W). 1990. 306 f. Dissertação (Mestrado em Oceanografia), CTG, UFPE, Recife.

ROHLF, F. J.; FISHER, D. L. Test for hierarclical structure in randon data sets. Systematical Zoology. (s.l.), v. 17, p. 107-412, 1968.

SANT'ANNA, E. M. E. Estrutura e biomassa da comunidade zooplanctônica da Bacia do Pina (Pernambuco, Brasil), relacionados com fatores ambientais. 1993. 195 f. Dissertação (Mestrado em Ciências da Engenharia Ambiental), Escola de Engenharia de São Carlos, USP, São Carlos.

SANTOS-FERNANDES, T. L. dos. Fitoplâncton do estuário do Rio Jaguaribe, (Itamaracá, Pernambuco, Brasil): Ecologia, densidade, biomassa e produção. 1997. 175f. Dissertação (Mestrado em Oceanografia), CTG, UFPE, Recife.

SILVA - CUNHA, M. da G.; ESKINAZI - LEÇA, E. Catálago das diatomáceas

(Bacillariophyceae) da Plataforma continental de Pernambuco. Recife: SUDENE, 1990. 318f.

Tropical Oceanography, Recife: v. 31, n. 2, p. 149-169, 2003. 
STRICKLAND, J. D. H., PARSONS, T. R. A pratical Handbook of seawater analyris. 2 ed. Bull. of Canada, Ottawa, n. 167, p. 207-211, 1972.

TETT, P.; WALNE, A. Observations and simulations of hidrography, nutrients and plankton in the Sourthen North Sea, Ophelia, Helsing, v. 42, p. 371- 416, Sept. 1995.

TRAVASSOS, P. E. P. F. Hidrologia e biomassa primária do fitoplâncton no Estuário do Rio Capibaribe, Recife - Pernambuco. 1991. 287 f. Dissertação (Mestrado em Oceanografia), CTG, UFPE, Recife.

TUNDISI, J. G. O plâncton estuarino. Contribuições avulsas. Instituto Oceanográfico. Série Oceanografia Biológica. São Paulo, v. 9, n. 1 - 22, 1970.

UNESCO. Determination of photosynthethic pigments in sea water. Rapor of scor/Unesco, working group 17 with meat from 40 to 6 june 1964. Paris, 1966. 69 f. (monography on Oceanography Methasology, 1).

UNESCO. International Oceanographic table opeat Britain wormly, 2, 1973, $141 \mathrm{f}$. 
Tabela 1 - Variação dos parâmetros hidrológicos nas estações estudadas.

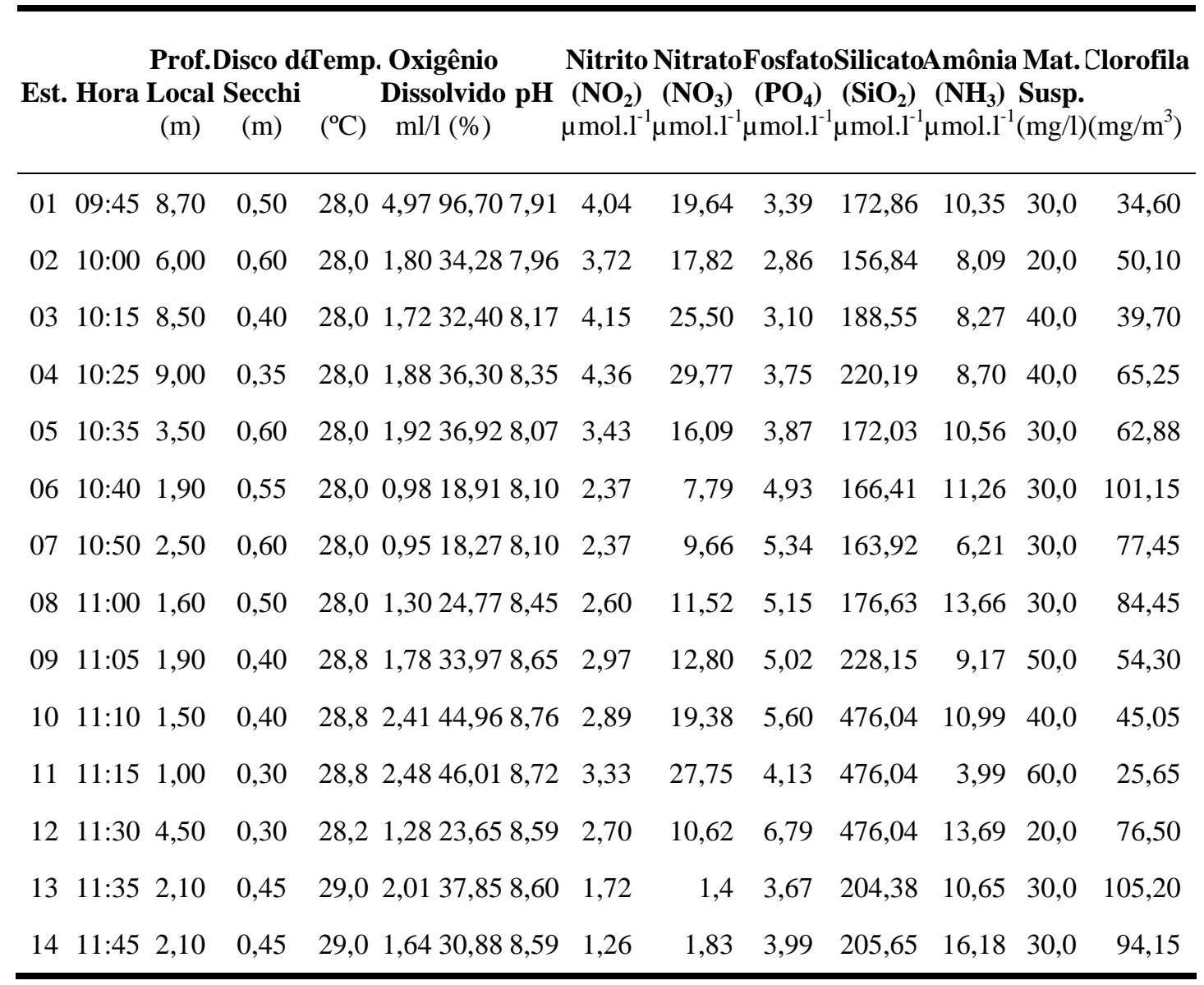


Tabela 2 - Variação da salinidade nas estações estudadas durante a baixa-mar.

\begin{tabular}{|c|c|c|c|}
\hline ESTAÇÃO & $\begin{array}{c}\text { HORA } \\
\text { DE } \\
\text { COLETA } \\
\end{array}$ & $\begin{array}{c}\text { PROFUNDIDADE } \\
\text { DE } \\
\text { COLETA } \\
\end{array}$ & $\begin{array}{l}\text { SALINIDADE } \\
\text { s.p. }\end{array}$ \\
\hline \multirow[t]{3}{*}{01} & 09:45 & Superfície & 10,77 \\
\hline & & Meio & 27,00 \\
\hline & & Fundo & 30,70 \\
\hline \multirow[t]{3}{*}{02} & $10: 00$ & Superfície & 7,00 \\
\hline & & Meio & 24,00 \\
\hline & & Fundo & 26,92 \\
\hline \multirow[t]{3}{*}{03} & $10: 15$ & Superfície & 4,84 \\
\hline & & Meio & 24,00 \\
\hline & & Fundo & 25,31 \\
\hline \multirow{3}{*}{04} & $10: 25$ & Superfície & 9,68 \\
\hline & & Meio & 24,00 \\
\hline & & Fundo & 25,83 \\
\hline \multirow[t]{3}{*}{05} & $10: 35$ & Superfície & 8,89 \\
\hline & & Meio & 16,00 \\
\hline & & Fundo & 17,49 \\
\hline \multirow[t]{3}{*}{06} & $10: 40$ & Superfície & 9,68 \\
\hline & & Meio & 11,00 \\
\hline & & Fundo & 9,68 \\
\hline \multirow[t]{3}{*}{07} & $10: 50$ & Superfície & 9,16 \\
\hline & & Meio & 8,00 \\
\hline & & Fundo & 9,95 \\
\hline \multirow[t]{3}{*}{08} & $11: 00$ & Superfície & 7,00 \\
\hline & & Meio & 7,00 \\
\hline & & Fundo & 7,53 \\
\hline \multirow[t]{3}{*}{09} & 11:05 & Superfície & 4,57 \\
\hline & & Meio & 5,00 \\
\hline & & Fundo & 6,20 \\
\hline \multirow[t]{3}{*}{10} & $11: 10$ & Superfície & 0,80 \\
\hline & & Meio & 5,00 \\
\hline & & Fundo & 6,47 \\
\hline \multirow[t]{3}{*}{11} & $11: 15$ & Superfície & 0,16 \\
\hline & & Meio & 0,00 \\
\hline & & Fundo & 0,80 \\
\hline \multirow[t]{3}{*}{12} & $11: 30$ & Superfície & 1,24 \\
\hline & & Meio & 4,00 \\
\hline & & Fundo & 1,63 \\
\hline \multirow[t]{3}{*}{13} & $11: 35$ & Superfície & 1,90 \\
\hline & & Meio & 6,00 \\
\hline & & Fundo & 1,90 \\
\hline \multirow[t]{3}{*}{14} & $11: 45$ & Superfície & 2,04 \\
\hline & & Meio & 4,00 \\
\hline & & Fundo & 2,11 \\
\hline
\end{tabular}

Tropical Oceanography, Recife: v. 31, n. 2, p. 149-169, 2003. 
Tabela 3 - Análise dos Componentes Principais no sistema estuarino da Bacia do Pina.

\begin{tabular}{l|c|c|c}
\hline \multicolumn{1}{c|}{ NOME } & $\begin{array}{c}\text { FATOR 1 } \\
\mathbf{( 3 6 , 4 0 \% )}\end{array}$ & $\begin{array}{c}\text { FATOR 2 } \\
\mathbf{( 3 3 , 2 4 \% )}\end{array}$ & $\begin{array}{c}\text { FATOR 3 } \\
\mathbf{( 1 0 , 4 4 \% )}\end{array}$ \\
\hline TRANSPARÊNCIA & 0.656 & 0.559 & 0.165 \\
TEMPERATURA & -0.814 & -0.072 & 0.549 \\
OXIGÊNIO DISSOLVIDO & 0.242 & -0.490 & 0.449 \\
pH & -0.954 & -0.175 & 0.028 \\
NITRITO & 0.602 & -0.716 & -0.218 \\
NITRATO & 0.320 & -0.889 & -0.216 \\
FOSFATO & -0.564 & 0.233 & -0.677 \\
SILICATO & -0.715 & -0.483 & -0.339 \\
AMÔNIA & -0.288 & 0.653 & -0.010 \\
MATERIAL EM SUSPENSÃO & -0.309 & -0.732 & 0.195 \\
CLOROFILA & -0.211 & 0.897 & -0.008 \\
SALINIDADE & 0.889 & 0.201 & -0.125 \\
\hline
\end{tabular}


Tropical Oceanography, Recife: v. 31, n. 2, 2003. 